\title{
KRAS NP_004976.2:p.G13C
}

National Cancer Institute

\section{Source}

National Cancer Institute. KRAS NP 004976.2:p.G13C. NCI Thesaurus. Code C98401.

A change in the amino acid residue at position 13 in the GTPase KRas protein where glycine has been replaced by cysteine. 